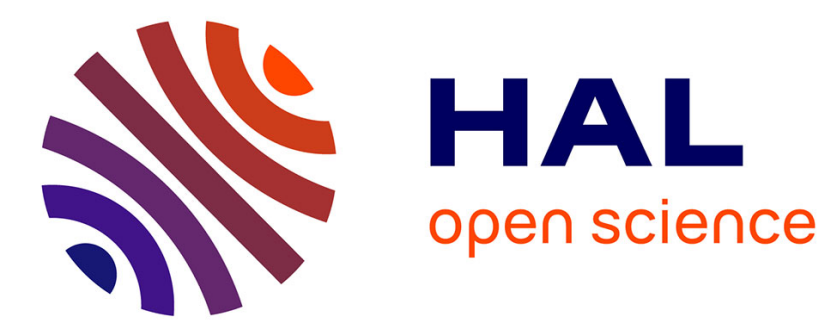

\title{
AcTinG: Accurate Freerider Tracking in Gossip
}

Sonia Ben Mokhtar, Jérémie Decouchant, Vivien Quéma

\section{To cite this version:}

Sonia Ben Mokhtar, Jérémie Decouchant, Vivien Quéma. AcTinG: Accurate Freerider Tracking in Gossip. Symposium on Reliable Distributed Systems (SRDS 2014), Oct 2014, Nara, Japan. 10.1109/SRDS.2014.12 . hal-01198451

\section{HAL Id: hal-01198451 https://hal.science/hal-01198451}

Submitted on 16 Sep 2015

HAL is a multi-disciplinary open access archive for the deposit and dissemination of scientific research documents, whether they are published or not. The documents may come from teaching and research institutions in France or abroad, or from public or private research centers.
L'archive ouverte pluridisciplinaire HAL, est destinée au dépôt et à la diffusion de documents scientifiques de niveau recherche, publiés ou non, émanant des établissements d'enseignement et de recherche français ou étrangers, des laboratoires publics ou privés. 


\section{AcTinG: Accurate Freerider Tracking in Gossip}

\author{
Sonia Ben Mokhtar \\ CNRS - LIRIS \\ sonia.benmokhtar@insa-lyon.fr
}

\author{
Jérémie Decouchant \\ Grenoble University \\ jeremie.decouchant@imag.fr
}

\author{
Vivien Quéma \\ Grenoble INP \\ vivien.quema@imag.fr
}

\begin{abstract}
Gossip-based content dissemination protocols are a scalable and cheap alternative to centralised content sharing systems. However, it is well known that these protocols suffer from rational nodes, i.e., nodes that aim at downloading the content without contributing their fair share to the system. While the problem of rational nodes that act individually has been well addressed in the literature, colluding rational nodes is still an open issue. Indeed, LiFTinG, the only existing gossip protocol addressing this issue, yields a high ratio of false positive accusations of correct nodes. In this paper, we propose AcTinG, a protocol that prevents rational collusions in gossip-based content dissemination protocols, while guaranteeing zero false positive accusations. We assess the performance of AcTinG on a testbed comprising 400 nodes running on 100 physical machines, and compare its behaviour in the presence of colluders against two state-of-the-art protocols: BAR Gossip that is the most robust protocol handling non-colluding rational nodes, and LiFTinG, the only existing gossip protocol that handles colluding nodes. The performance evaluation shows that AcTinG is able to deliver all messages despite the presence of colluders, whereas both LiFTinG and BAR Gossip suffer heavy message loss. It also shows that AcTinG is resilient to massive churn. Finally, using simulations involving up to a million nodes, we show that AcTinG exhibits similar scalability properties as standard gossip-based dissemination protocols.
\end{abstract}

\section{INTRODUCTION}

It is well known that content sharing applications account for a large proportion of traffic over the Internet. The most popular of these applications include collaborative downloading (e.g., BitTorrent) and peer-to-peer live streaming (e.g., $\mathrm{P} 2 \mathrm{PLive})$. Relying on the $\mathrm{P} 2 \mathrm{P}$ paradigm offers robustness to failures, scalability up to hundreds of thousands of nodes, and adaptability. Indeed, P2P systems can handle massive node arrival/departure and are highly resilient to churn. From the point of view of content providers, relying on a P2P system allows shifting cost (e.g., bandwidth) to clients, and avoids the need for maintaining dedicated servers.

A major problem that face large scale P2P systems deployed on the public domain is the existence of rational nodes, i.e., nodes that aim at receiving content without contributing their fair share, by forwarding it to others. Existing studies have shown that the presence of even a small portion of rational nodes significantly degrades the system performance [1]-[5]. This is why a number of protocols have been devised in the last decade to deal with the problem of rational nodes in collaborative systems, (e.g., rational resilient live streaming [6]-[8], spam filtering content dissemination [9] and Nparty transfer [10]). All these protocols provide incentives that encourage/force rational nodes to participate in the system.
However, apart from the protocol presented in [8], all the existing solutions work under the assumption that rational nodes do not collude. This problem though has been demonstrated to be a reality in existing file sharing applications [11]. Handling colluding nodes is a difficult problem provided that colluders generally perform unobservable actions from the point of view of the collaborative protocol [12], which makes their deviations difficult to deter. For example, a group of colluders could be a group of nodes that collaborate to exchange content between each other "off the record" (e.g., using the silent broadcast protocol described in [12]). Such colluders do not share with nodes not belonging to the group the content they receive off the record, thus harming the protocol.

To the best of our knowledge, the only gossip-based content dissemination protocol trying to prevent collusions is the LiFTinG protocol [8]. In this protocol, nodes log their interactions with other nodes and perform distributed audits of each others logs. In order to be cost effective, this protocol relies on cryptography-free procedures and statistical analysis of these logs. For instance, a node is suspected of colluding with another node if the frequency of its interactions with the latter is greater than an expected average. Unfortunately, as analysed by the authors themselves, due to their statistical nature and to message losses, the mechanisms implemented in LiFTinG do not allow to catch all rational collusions (false negatives), and may even lead to wrong exclusions of correct nodes (false positives). Experiments that we performed, and that are described in Section VI-B, confirm this result and further show that, for instance, when $30 \%$ of nodes collude (either in a large group or in small collusion groups), correct nodes observe $25 \%$ of message losses.

The challenge we embrace in this paper is the design of a rational-resilient content dissemination protocol that prevents collusions to occur and that does not wrongfully exclude correct nodes. An observation one can start with is: a colluding behaviour can be considered as a Byzantine behaviour [13]. A legitimate question is thus to know whether it is possible to rely on existing techniques for Byzantine fault tolerance and Byzantine fault detection, such as Nysiad [14], PeerReview [15], Accountable Virtual Machines [16], Trinc [17], or A2M [18]? The answer is No. The reason is that these generic solutions for Byzantine fault tolerance and detection either assume a limited proportion of faulty nodes, or the existence of trusted nodes or hardware. Instead, we assume in this paper that all nodes can be rational, and we do not rely on any trusted entity, whether software or hardware. 
In this paper, we present AcTinG a content dissemination protocol that tolerates an unlimited number of (possibly colluding) rational nodes, while guaranteeing that no correct node is ever expelled, and that all rational deviations are eventually detected. To reach this objective, we adopt a different approach than the one used in the LiFTinG protocol: rather than trying to detect collusions a-posteriori, we built AcTinG in such a way that it is not in the interest of nodes to collude. We analyse each step of the protocol and describe the incentives that force rational (possibly colluding) nodes to stick to the protocol. We perform a performance evaluation of AcTinG. Its performance is compared with two protocols: BAR Gossip, the state-of-theart gossip protocol that is able to handle non-colluding rational nodes and LiFTinG, the state-of-the-art gossip protocol that is able to handle colluding nodes. We implement a streaming application that we deploy on top of the three protocols. We deploy 400 nodes on one hundred physical machines and show that AcTinG is able to deliver the entire stream despite the presence of colluders, whereas LiFTinG and BAR Gossip, both suffer heavy message losses. We also show that AcTinG is resilient to churn, and using complementary simulations involving up to a million nodes, that it is scalable: it yields a logarithmic growth of memory and bandwidth consumption, comparable to standard gossip based protocols [19].

The rest of the paper is structured as follows. Section II describes our system model. Section III introduces the core ideas of AcTinG. Section IV provides a detailed presentation of AcTinG. Section V discusses its resilience to (colluding) rational nodes. Section VI presents a detailed performance evaluation. Section VII reviews the related works. Section VIII concludes the paper.

\section{SySTEM Model}

We consider a system with $N$ nodes, which are uniquely identified, e.g., using a hash value of their IP address. We assume that nodes can join and leave the system (gently or by crashing) at any time. We consider two classes of nodes: correct nodes and rational nodes. Correct nodes follow the protocol. Rational nodes are defined as in [7] extended with the notion of collusion: they aim at getting the content (i.e., missing the lowest possible number of updates) at the lowest possible overhead in terms of bandwidth consumption. This means that rational nodes would deviate in any sort from the protocol, possibly by colluding with each other, as long as the deviation saves their resources while not impacting the quality of the content they are getting.

Specifically, the benefit of colluding rational nodes can be represented along the following axes:

1) (Stream Quality) Receiving as much as possible (possibly, all) stream updates,

2) (Communication) Sending as little as possible (possibly, none) stream updates or protocol messages to nodes not belonging to their coalition,

3) (Computation) Performing as little as possible computations for other nodes.
Colluding rational nodes would typically exchange updates off the record, and, in order to save bandwidth, would not share the updates they obtained secretly with nodes outside their group. It is important to note that rational nodes are risk averse, i.e., they never deviate from the protocol if there is any risk of being evicted from the system. This assumption is commonly used in BAR systems [20]. Furthermore, this assumption is particularly relevant in our context as we use accountability techniques to deter faults and accuse nodes (as described in the following section). In this context, when a fault is detected, a proof of misbehaviour is produced, which can convince any correct node in the system of the necessity of evicting the misbehaving node. As eviction corresponds to an infinite penalty, no benefit is worth taking such risk. We also suppose that rational nodes join and remain in the system for a long time and seek a long-term benefit.

We refer to the source as the node that is disseminating a given content. We assume that each content is disseminated from a single source at a time but our principles can be easily applied to systems where the content is disseminated from multiple sources at the same time. We assume that all nodes but the source may be rational, or experience failures, and may organise themselves in colluding groups of arbitrary sizes. Classical fault-tolerance techniques (e.g., [21]) can relax the assumption that the source does not fail.

We assume that the network allows every pair of nodes to exchange messages, and that they are eventually received if retransmitted sufficiently often. We also assume that hash functions are collision resistant and that cryptographic primitives cannot be forged. We assume that nodes are provided a pair of asymmetric keys, and denote a message $m$ signed by a node $i$ using its private key as $(m)_{\sigma(i)}$.

As in [7] and [22], we assume that nodes maintain clocks synchronised within $\delta$ seconds, and we structure time as a sequence of rounds in which nodes exchange updates. We assume that nodes have a secure log that is used to check their correctness through its analysis. A secure $\log$ is a $\log$ that is tamper evident and append only. Many systems recently defined variants of secure logs among which [15]-[18]. We build on the secure log presented in [15].

\section{Protocol Overview}

We present AcTinG, a gossip-based dissemination protocol that guarantees the following two properties: (i) a correct node is never expelled, and (ii) a rational node that deviates from the protocol in a way that impacts the performance of correct nodes is eventually suspected by all correct nodes. In the remainder of this section, we describe the principles of AcTing that allow us to guarantee the above two properties. Protocol details are then presented in Section IV.

Figure 1 shows an overview of our protocol. In this figure, the source node $s$, which is the node from which the dissemination originates, cuts the content into chunks that we call updates. It then periodically disseminates these updates to a set of nodes (arrows 1 in the figure). To join this content dissemination session, a new node ( $p_{n}$ in the figure) needs 


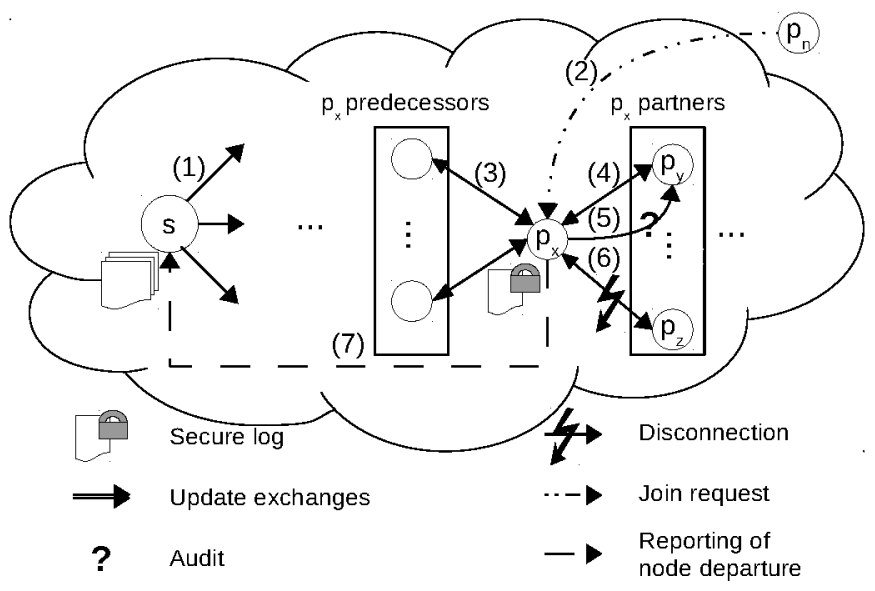

Fig. 1. Overview of AcTinG.

to know a node that is already part of it, as described in Section IV-A (arrow 2 in the figure). In the middle of the figure, a node $p_{x}$, which characterises any node in the system except the source, has a set of nodes that it has selected as partners (depicted on its right side in the figure). Further, $p_{x}$ has a set of nodes that selected it as a partner (depicted on its left side), to which we refer as $p_{x}$ 's predecessors. Periodically, $p_{x}$ has to share with its partners (arrow 4 in the figure) and with its predecessors (arrow 3 in the figure) the updates it received. In order to maximise the quality of the content it receives, $p_{x}$ may be tempted to (1) act rationally by receiving updates and not sharing them with its partners, or predecessors, and (2) collude with other nodes in the system (not necessarily its partners or predecessors) to get updates off the record without sharing them with anyone else. To avoid these temptations, the core idea underlying AcTinG is to make nodes accountable for their actions. Specifically, each node in AcTinG logs in a secure $\log$ its interactions with other nodes in the system, including the identifiers of the updates it received. Because any node can verify the information in the $\log$ of a node it is interacting with, the latter will be obliged to send to its partners the updates it has, and to receive the updates it is missing. Consequently, no node will have an interest in behaving rationally or forming collusions. Indeed, assume that node $p_{x}$ colludes with another node to receive an update $u$ off the record. Node $p_{x}$ will not be able to record update $u$ in its $\log$ (because the exchange was unofficial; we explain later how it is done). The good news for node $p_{x}$ is that it does not have to forward $u$ to other nodes because $u$ does not appear in its log. The problem is that the next time a correct node having $u$ in its $\log$ will interact with node $p_{x}$, it will send update $u$ to $p_{x}$. Consequently, $p_{x}$ will eventually have to forward $u$, and thus will have wasted its bandwidth, because it will have received $u$ twice (off the record and from a correct node).

This core idea raises several questions and challenges that we answer in the remainder of this section.

"What if $p_{x}$ chooses only colluders as partners with which it will interact with in the near future?'. This way, $p_{x}$ could accept updates and arrange with its future partners so as they do not audit its $\log$, or so they do not send it updates it already received unofficially. Our protocol deals with this issue by forcing nodes to (periodically) establish random, yet deterministically verifiable partnerships as presented in Section IV-B. Specifically, each time a node $p_{x}$ has to change its partners, it computes their identifier using a pseudo random generation function seeded with a deterministically computed seed. As such, nodes that will audit its $\log$ will be able to verify the legitimacy of the partners that it has selected.

"What if a node, $p_{x}$, maintains many (correct) logs?". For instance, $p_{x}$ could have a $\log$ in which an update $u$ appears, which it will show to nodes who already have $u$ (to avoid sending it to them), and another log in which the same update does not appear, which will be presented to nodes that do not have $u$ (to avoid having to send it to them). This problem is known as equivocation, i.e., the ability to make conflicting statements to different participants [17]. We deal with this issue by forcing nodes to audit their partners' logs at the beginning of each new partnership (arrow 5 in the figure). This audit verifies the consistency of the $\log$ of a node as a whole as presented in Section IV-C.

"Isn't this periodic exchange of logs a performance overkill?". It is not necessary to audit the logs of nodes each time two nodes exchange updates. Indeed, we build on the assumption that colluders, and rational nodes in general, are risk averse. Hence, it is enough to ensure that for each step of the protocol, a deviation has a high probability to be detected in the near future, in order to make sure that rational nodes will not deviate. Consequently, instead of performing audits each time nodes communicate, audits are triggered in a random yet verifiable manner. Indeed, audits (from the point of view of audited nodes) must not be predictable, because rational nodes would seize an opportunity to deviate undetected if they could predict them. Yet they must be verifiable (from the point of view of nodes performing them), because rational nodes have to be forced to trigger this procedure. To reach this objective, a node that starts a new partnership with a node performs a deterministic computation that results in a boolean telling it whether it should audit its partner or not.

"What if rational nodes decide not to answer to correct nodes to avoid trading updates, or being audited?". There are many reasons why a rational node may be tempted not to answer to a request from a correct node. This could, for instance, preserve it from sending its log and being audited as a result (arrow 6 in the figure). This type of misbehaviour is known as omission failures. We deal with this problem using a mechanism where unresponsive nodes are eventually suspected by all correct nodes, which stop interacting with them (as described in Section IV-A). As it is not in the interest of rational nodes to be isolated in the system, a rational node in AcTinG will answer all correct node requests. To avoid correct nodes to be expelled from the system because one of their message has been lost or delayed, we allow suspicions to be released, e.g., if the missing message eventually arrives. Similarly, rational nodes may be tempted to wrongly suspect correct nodes of omission failure, by claiming that they did not send a given message to them, as it is the only reason why a 
node can skip mandatory interactions. We avoid this deviation by overcharging the sending of suspicion messages in such a way that it is more costly to suspect a node of omission failure than to effectively interact with it. As such, nodes would suspect other nodes of omission failures only if they are really missing a given message. Instead, if a node effectively left the system (assume node $p_{z}$ in the figure), its predecessors (among which, node $p_{x}$ in the figure) contact $p_{z}$ 's partners to collect evidence about the effective unresponsiveness of $p_{z}$ (as described in Section IV-A). Then, $p_{x}$ sends this evidence to the source node (arrow 7 in the figure), which eventually updates the membership list, and will also inform its partners during future exchanges.

Summarising, our protocol builds on accountability techniques, and on a set of mechanisms to provide incentives to rational, possibly colluding, nodes to stick to the protocol. Specifically, to avoid nodes from selecting their partners, our protocol relies on random yet verifiable partnerships. To be efficient it relies on random yet verifiable audits. To discourage rational nodes from being falsely unresponsive, our protocol handles omission failures. Finally, to discourage nodes from wrongly suspecting their partners our protocol associates an extra cost with suspicion messages.

\section{Protocol Details}

We have presented the principles of AcTinG in the previous section. In this section, we detail the steps of the protocol.

In a nutshell, AcTinG divides time in rounds. At each round the source disseminates new updates, which come to expiration after RTE rounds, to a small set of randomly chosen nodes. To get updates, each node initiates and maintains partnerships with other nodes with whom it exchanges updates at each round. The partners are selected using a pseudo-random number generator function, i.e., PRNG, seeded deterministically (e.g., with the node's public key concatenated with the round number). At the beginning of a round, each node contacts all of its partners in order to propose updates to them and to request updates from them. Every Period rounds, each node updates its set of partners. Each time a node starts a new partnership with a node, the two nodes audit each others' $\log$ with a given probability. The membership is managed in a distributed manner by nodes who periodically inform the source of the arrival and the departure of nodes. Yet, it is the responsibility of the source to disseminate an updated list of alive nodes every Epoch rounds.

The remainder of this section describes the sub protocols constituting AcTinG in detail, as follows. First, we present the membership protocol (Section IV-A), which allows dealing with new nodes joining the system, nodes leaving it and unresponsive nodes. Then, we present the partnership management (Section IV-B), the audit (Section IV-C) and the update exchange protocols (Section IV-D), which allow handling the partnerships between nodes, auditing their logs and exchanging updates between partners, respectively.

\section{A. Membership protocol}

The membership protocol handles the arrival and the departure of nodes as well as the management of the membership list. Our membership protocol is fully distributed, rational resilient, and handles massive nodes arrival and departure.

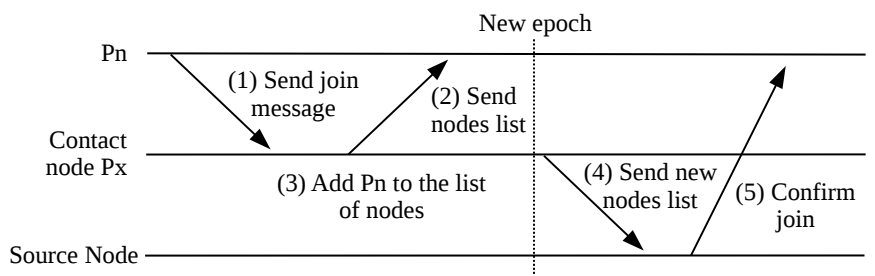

Fig. 2. Arrival of a new node.

Node arrival: The arrival of a new node follows the sequence of messages depicted in Figure 2. In this figure, we assume that node $p_{n}$, which would like to join a given content dissemination session, has installed the AcTinG software. This means that $p_{n}$ has an empty secure log with the related security primitives. We also assume that $p_{n}$ knows an entry point in the system, say $p_{x}$, which we call the contact node of $p_{n}$. To join a content dissemination session, $p_{n}$ sends a join request to $p_{x}$ (step (1) in the diagram). The latter replies with the list of active nodes of the current epoch (step (2) in the diagram). Using this list, $p_{n}$ computes its list of new partners using the PRNG function as described in Section IV-B and contacts them to start receiving the content. At the beginning of a new round, each node, including node $p_{x}$ informs the source of the arrival of new members that have contacted it (step (4)). Using theses messages, the source confirms to the new members their integration in the system and updates the membership list (step (5)).

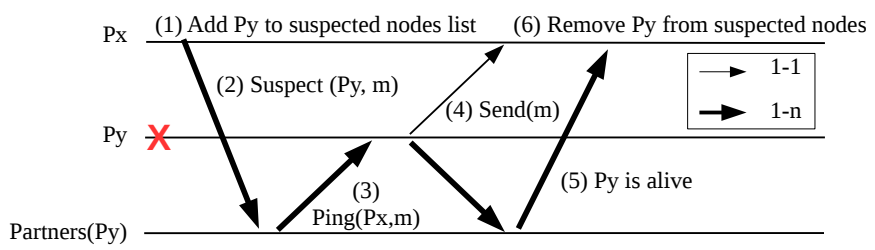

Fig. 3. Handling of an omission failure.

Node departure and omission failures: If a node $p_{x}$ is expecting a message from one of its partners $p_{y}$ for too long ${ }^{1}$, it suspects $p_{y}$ of omission failure as depicted in the diagram of Figure 3. Specifically, $p_{x}$ adds $p_{y}$ in its local list of suspected nodes (step (1) in the figure) and sends a suspicion message to the other partners of $p_{y}$ (step (2)). This message includes the type of message that $p_{x}$ is expecting from $p_{y}$. Then, each of $p_{y}$ 's partners pings $p_{y}$ (step (3)). If $p_{y}$ is alive, it replies to both its partners and $p_{x}$ with the missing message (step (4)). After a given time slot, each of $p_{y}$ 's partners replies to $p_{x}$ with a signed message certifying whether $p_{y}$ responded to the ping message or not (step (5)). Using this message, $p_{x}$ either

\footnotetext{
${ }^{1}$ Delays for node suspicion are configured in an implementation dependent manner
} 
removes $p_{y}$ from its list of suspected nodes if $p_{y}$ replied (step (6)) or sends an eviction message to the source including the messages received from $p_{y}$ 's partners.

To be sure that a rational node will never suspect a correct node, in order to avoid initiating or accepting an interaction, we make the cost of sending a suspicion message higher than the cost of a normal interaction. Hence, unless it is a real suspicion, a node will never suspect another node.

Membership list update: Periodically, nodes that served as contact nodes for others send their list of new nodes to the source. Furthermore, nodes that hold an evidence of the departure of one partner send it to the source. The latter updates the membership list and sends it, at the beginning of each epoch, to the nodes along with the content. In order to fasten the removal of dead nodes from the membership list, an optimisation consists in letting the source disseminate the list of dead nodes at the beginning of each round along with the stream, instead of waiting the following epoch. As soon as a node receives these incremental updates from the source, it removes the corresponding nodes from its list of alive nodes, which avoids selecting them when new partnerships have to be established before the new epoch. In order to preserve nodes from the massive arrival of new nodes, which may consume their bandwidth, we adopt the optimisation defined in [22], which allows splitting the load between the older nodes and the new ones. Specifically, this optimisation prevents new nodes from establishing too many partnerships with older nodes.

\section{B. Partnership management}

Each node $p_{x}$ maintains partnerships with $f$ other nodes, which are selected with the PRNG function seeded with a deterministically computed seed (e.g., $p_{x}$ 's public key concatenated with the round number) among the non-suspected nodes of the last membership list. This process is depicted in the diagram of Figure 4. If a selected node is not responding, node $p_{x}$ has to propagate a suspicion, and once it is confirmed, $p_{x}$ is allowed to find a new partner. Every Period rounds, a node $p_{x}$ breaks the $f$ partnerships it initiated, without informing its partners which know when the partnerships are supposed to end. A node having an identifier $i d$ will change its partnerships during round $r$ if $(i d+r) \bmod$ Period $=0$. To initiate a new partnership with a node $p_{y}$, node $p_{x}$ sends an association request to $p_{y}$ (step (2) in the diagram).

At the beginning of a partnership, a node $p_{x}$ may trigger an in-depth audit of its new partner $p_{y}$ (step (4) in the diagram), by contacting the partners $p_{y}$ had in the RTE previous rounds, and asking them to return their own log of the last RTE rounds including the current round (step (5) in the diagram). To reduce the cost of the protocol, nodes perform these audits in a random manner, i.e., each time they are in a position to perform an audit, they flip a coin and decide whether they should audit their partner or not. Nevertheless, to avoid that rational nodes hide behind this randomness to avoid auditing their partners, we make this randomness verifiable. Towards this purpose, we use the secure log authenticators, which are signed messages computed from the node's log as detailed in
Section IV-C. These values are unpredictable as they depend on the current state of a node's log. Specifically, each time a node $p_{x}$ is in a position to perform an audit of a new partner $p_{y}$, it computes the hash of its public key concatenated with the public key of $p_{y}$ and the round number. The value of this hash modulo 100 gives a number that $p_{x}$ uses to decide whether it should audit its new partner. For instance, if the probability of auditing a node fixed by the protocol is $30 \%, p_{x}$ audits $p_{y}$ if the result of the modulo function is between 0 and 29. Node $p_{x}$ further $\operatorname{logs}$ the authenticators it used to compute the value of this boolean, in order to justify, in future audits, the reason why it performed or did not perform the audit of $p_{y}$. If the audit must take place, $p_{x}$ contacts $p_{y}$ 's partners, and asks for their logs.

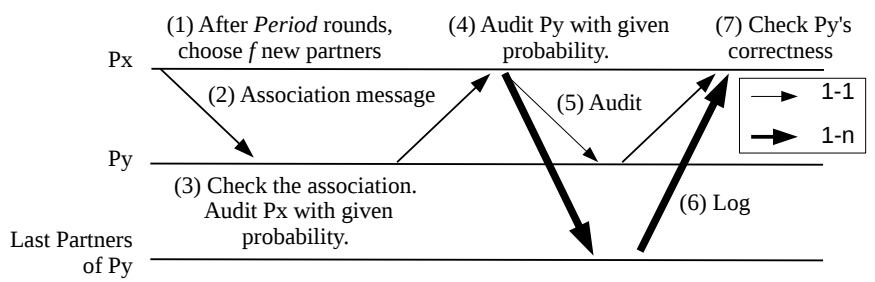

Fig. 4. Establishment of new associations between nodes, which may imply audits.

\section{Audit protocol}

In our protocol, the secure log is used to keep track of the communication a node had with other nodes in the system. Specifically, each entry in the $\log$ of a node $A$ corresponds to a message sent (resp. received) by $A$ to (resp. from) another node $B$. A $\log$ entry $e_{i}$ is of the form $e_{i}=\left(\right.$ seqno $\left._{i}, h_{i}, c_{i}\right)$ where seqno $_{i}$ is a monotonically increasing sequence number, $h_{i}$ is a hash value linked with the previous entries in the $\log$ and $c_{i}$ is a type-specific content, which may include the message sent (resp. received) by $A$ as well as other information such as authenticators (as defined below). The value of $h_{i}$ is computed as follows: $h_{i}=H\left(h_{i-1} \|\right.$ seqno $\left._{i} \| H\left(c_{i}\right)\right)$, where $h_{0}=0, H$ is a hash function and $\|$ stands for concatenation.

Each time a $\log$ entry $e_{i}$ is added to the $\log$ of a node $A$, an authenticator $\alpha_{i}$ is generated. This authenticator, which is a signed message $\alpha_{i}=\left(\text { seqno }_{i}, h_{i}\right)_{\sigma(A)}$, states that $A$ has a $\log$ entry $e_{i}$ with a corresponding hash $h_{i}$. By sending the authenticator $\alpha_{i}$ to a node $\mathrm{B}$, A commits to having logged the entry $e_{i}$ and to the content of its $\log$ before $e_{i}$. Any node that receives $\alpha_{i}$ can use it to inspect $e_{i}$ and all the entries preceding $e_{i}$ in the $\log$ of $A$. Upon reception of a $\log$, any node is able to recompute the hash values it contains, according to the log entries, and thus to check their validity. In addition, a log entry for a received message must include a matching authenticator, implying that a node cannot invent an entry for a message it did not receive. These two properties make the secure logs tamper-evident and append only.

As described in the partnership management protocol, when node $p_{x}$ must audit node $p_{y}$, it asks $p_{y}$ 's partners to return their logs. Upon reception of these $\operatorname{logs}$, node $p_{x}$ verifies:

(i) the consistency of the logs, by recomputing the recursive hash values associated to $\log$ entries, 
(ii) the presence of the exchanges $p_{y}$ was supposed to initiate, (iii) that $p_{y}$ declared the updates it was supposed to receive from the source, if $p_{y}$ was supposed to interact with the source, (iv) that the exchanges correspond to a correct execution of the protocol, i.e., that $p_{y}$ proposed to all its partners all the updates that appear in its $\log$, that $p_{y}$ requested from its partners all the updates it was missing, that $p_{y}$ served to its partner all the updates they were requesting and that $p_{y}$ logged all the identifiers of the updates it received,

(v) that $p_{y}$ suspected all its partners that did not follow a given step of the protocol as prescribed by the omission failure protocol,

(vi) that $p_{y}$ audited all the partners it was supposed to audit. As any other node, the source also maintains partnerships and regularly changes its partners, i.e., the nodes it serves. The source follows the partnership management and the updates exchange protocols, except that it does not send any log and it is not audited by nodes ${ }^{2}$. This forces the nodes to log the identifiers of the updates they received from the source, as they are deterministically chosen among the epoch membership list, which is known by all nodes. Hence, any node can check that the received updates were correctly declared. As the serving rate of the source is constant, the identifier of the updates that are released at each round are also known.

\section{Update exchanges}

At the beginning of each round and for the duration of their partnership, two partners $p_{x}$ and $p_{y}$ exchange updates as depicted in Figure 5. Specifically, node $p_{x}$ (resp. $p_{y}$ ) starts the exchange by generating a proposition message containing the identifiers of all the updates that appear in its log and that did not expire yet. Node $p_{x}$ (resp. $p_{y}$ ) logs this proposition message in its $\log$ and generates the corresponding authenticator. Then, $p_{x}$ (resp. $p_{y}$ ) sends the proposition message along with the corresponding authenticator to $p_{y}$. Upon reception of the proposition message, which it logs, node $p_{y}$ (resp. $p_{x}$ ) selects the updates it is missing and replies to $p_{x}$ (resp. $p_{y}$ ) with an update request. The update request is logged at the two parties. Finally, $p_{x}$ (resp. $p_{y}$ ) serves the missing updates, and logs the serve message. Each partner then terminates the exchange by logging the identifiers of the updates it received, in its log. The nodes will then propagate the received updates during the following rounds, because we cannot ensure that nodes will immediately share them.

\section{RISK VERSUS GAIN ANALYSIS}

The aim of this section is to demonstrate that rational nodes will not collude with their partners, because audits will detect deviations with a high probability, and because the estimated gain of collective deviations is low. Complementary to this analysis is a proof that the protocol is a Nash equilibrium. This proof lists all the protocol steps and possible rational deviations and proves that rational nodes do not have any interest in deviating from the protocol whether individually

\footnotetext{
${ }^{2} \mathrm{We}$ recall that the source is assumed to be a correct node.
}

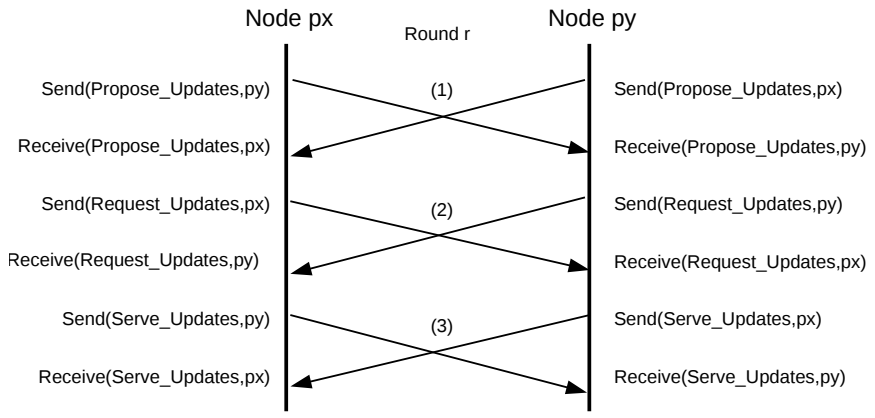

Fig. 5. Update exchanges between nodes.

or as a group. Due to the lack of space, this proof is available in the companion technical report [23].

We first evaluate the risk that two colluding partners take by deviating, for example when interacting as prescribed by the protocol, but without logging the updates they exchange. Specifically, consider two partners $p_{x}$ and $p_{y}$ that decide to collude. Assume $p_{x}$ holds update $u$. To help $p_{y}$ saving bandwidth in future rounds, $p_{y}$ sends a proposition message to $p_{x}$ that does not contain $u$, but logs that it has proposed $u$. As such, the $\operatorname{logs}$ of $p_{x}$ and $p_{y}$ appear correct if audited separately as $p_{x}$ can not be blamed of not requesting $u$ (as the official proposition sent by $p_{y}$ does not contain $u$ ) and $p_{y}$ can not be blamed of not proposing $u$ as it appears in his log that he has done so. We define the risk as the probability that such a deviation is deterred by an audit.

Let us compute this risk. If any of the two colluding nodes is audited during the time where the exchange is contained in their logs, they will be discovered. Let us consider a system of $N$ nodes, where $C$ nodes are part of a single colluding group. A node's log contains the entries of the last $R T E$ rounds. A participating node initiates $f$ partnerships with other nodes, which are changed after Period rounds. Let $P_{\text {audit }}$ the probability that a node audits each of its new partners. When establishing a new partnership, a rational node is not audited if its new partner is colluding with it (which happens with probability $\frac{C}{N}$ ), or if the new partner is correct but the protocol prescribes not to perform the audit. On average, each of the two nodes interacts with $\frac{2 \times f \times R T E}{\text { Period }}$ partners during the time the deviation is visible. Finally, we obtain that the risk a deviation is detected is equal to: $\left(1-\left(\frac{C}{N}+\left(1-\frac{C}{N}\right) \times\left(1-P_{\text {audit }}\right)\right)^{\frac{2 . f . R T E}{\text { Period }}}\right)^{2}$

Let us now compute the gain of performing the above deviation. To do this we need to compute the number of interactions that a rational node may have with correct nodes that do not hold the update $u$ after receiving it from its colluding partner, i.e., correct nodes to which the rational node would have had to send $u$ if it has received it officially from its colluding partner. To do so, we use the algorithm of Figure 6. The principle of this algorithm is that during each of the $R T E$ rounds that follow the round at which the deviation occurred, $2 * f$ interactions happen. Each of these interactions, has a probability $\frac{C}{N}$ to involve another colluding node. When it is not the case, this other node owns the missing update with 
a probability that depends on the number of rounds elapsed since its release by the source. We evaluate this probability using another algorithm, detailed in the companion technical report [23]. When the rational node receives the update from a correct node, it will have to share it with its future partners.

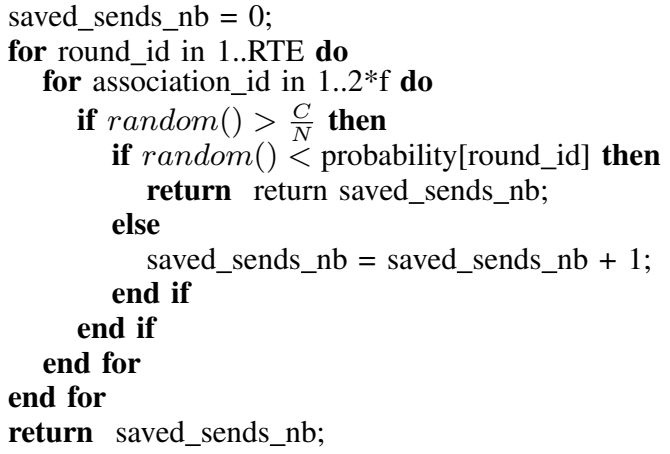

Fig. 6. Pseudocode of the algorithm used to estimate the number of times a colluding node avoids to send an update.

Using the average of the outputs of this algorithm, we can compute the proportion of interactions in which an update will not be sent by rational nodes. To obtain the long term gain, we multiply this proportion by the probability that a rational node has to meet an accomplice to be able to execute this deviation, which is $\frac{C}{N}$.

Computing the risk, and the gain, with the values of the parameters used in the protocol and further described in the following section, we obtain that the risk two colluding nodes take is equal to $60 \%$, and the long term gain of the associated deviation is equal to $3 \%$. Thus, rational nodes are exposed with a high risk each time they execute the deviation, and can only hope for a very small benefit. As a result, we conclude that rational nodes will not collude with their partners to exchange updates off the record. As said above, a complete analysis of the incentives provided by the protocol can be found in [23]. Note that nodes can still collude silently with nodes that are not their partners. Yet, it they do so, they are still obliged to execute the protocol correctly, i.e., request updates they do not officially hold and propose updates they officially hold to correct nodes. Hence, their collusion will not have any impact on the quality of the stream perceived by correct nodes.

\section{PERFORMANCE EVALUATION}

In this section, we present the performance evaluation of the AcTinG protocol. We start by introducing our methodology (Section VI-A). Then, we compare the impact of colluders on AcTinG, BAR Gossip, and LiFTinG (Section VI-B). We choose BAR Gossip as it is the most robust rational resilient content dissemination protocol that has been proposed so far and LiFTing as it is the only state-of-the-art content dissemination protocol that handles colluders. We then assess the bandwidth consumption of AcTinG (Section VI-C), its performance in the case of massive node departure (Section VI-D) and its scalability in terms of memory and bandwidth con- sumption using simulations involving up to a million nodes (Section VI-E).

Overall, our evaluation draws the following conclusions: In a real deployment involving 400 nodes and in presence of colluders, correct nodes using AcTinG do not experience any degradation in the quality of the content they receive while those using BAR Gossip and LiFTinG experience heavy message loss in presence of colluders independently from their organisation (whether in small or larger groups). On the other hand, we show that nodes that decide to collude in AcTinG experience a heavy overhead, which discourages them from staying in the coalition. Moreover, we show that AcTinG bandwidth consumption is reasonable and that AcTinG is resilient to massive node departure. Finally, we show that AcTinG is scalable as simulations involving up to a million nodes exhibit that both the bandwidth and memory consumptions of AcTinG follow a logarithmic growth in the number of nodes. However, we acknowledge that the source may become a bottleneck as the number of nodes increase, as it periodically receive notifications. Solving this issue is classically done by using a tracker, i.e., a centralised server that handles membership, as in the FlightPath protocol [22], which could easily be integrated in our system. The tracker could even be replicated using classical fault-tolerance techniques (e.g., [21]).

\section{A. Methodology and Parameter Setting}

To assess the performance of AcTinG, BAR Gossip and LiFTinG, we used them to implement three video live streaming applications. In these applications, a source node, selected randomly, diffuses a video stream at a rate of $300 \mathrm{kbps}$, during 5 minutes, and proposes each update to 5 random nodes. Updates are then disseminated using either AcTinG, BAR Gossip or LiFTinG, respectively. In order to provide a fair comparison, we implemented the three streaming applications in Java using the same code base. We deployed the three applications in 400 nodes running in one hundred physical machines of the Grid5000 cluster, interconnected with a $1 \mathrm{~Gb} / \mathrm{s}$ network that we limited to $1 \mathrm{Mb} / \mathrm{s}$. Each machine is composed of an Intel Xeon L5420 processor clocked at $2.5 \mathrm{GHz}$ with $32 \mathrm{~GB}$ of RAM. In the three applications, to provide further tolerance to message loss (combined with retransmissions), the source groups packets in windows of 40 packets, including 4 FEC ${ }^{3}$ coded packets.

The duration of one round is set to one second, and updates are released 10 seconds before being consumed by the nodes media player. Note that nodes dynamically adapt the number of their partners according to the size of the membership list: each node establishes $\left[\frac{\ln (N)}{2}\right]$ partnerships that it maintains for a duration of five rounds. For instance, in the fault free case, with $N=400$, each node has 3 partners. At the beginning of each partnership, nodes performed audits with a probability of $5 \%$, which, as we show in Section V, allows the system to detect deviations with a probability of $60 \%$ when up to $10 \%$ of the audience colludes in a single group. The

\footnotetext{
${ }^{3} \mathrm{FEC}$ stands for Forward Error Correction.
} 
cryptographic primitives consisted in a 1024-bit RSA signature and a SHA-1 hash.

\section{B. Impact of Colluders}

In this section, we experimentally study the impact of colluders on the BAR Gossip, LiFTinG, and AcTinG protocols. We implemented colluders from the code base of correct nodes in each protocol as follows. Colluders exchange unofficially among each other all the stream updates they received from correct nodes. Furthermore, colluders execute all the possible undetectable rational deviations that exist in the underlying protocol. For instance, in BAR Gossip, colluders never take part of the optimistic push protocol, which allows nodes to altruistically push updates to other nodes. Similarly, in LiFTinG, colluders do not audit the logs of other nodes and do not reply to messages sent by other nodes asking them to assess the behaviour of their previous partners unless the considered partner is among the group. As a result, correct nodes will be blamed by their correct auditors. In this situation the system administrator has two choices: (1) adjust the detection threshold to avoid false positives (by decreasing its value), which opens the doors to colluders for freeriding or (2) adjust the detection threshold to detect colluders (by increasing its value), which results in very high values of false positive accusations. In this experiment, we considered the first situation. A complementary experiment showed that in the second situation, adjusting the threshold to exclude $20 \%$ of colluders incurred the exclusion of $43 \%$ of correct nodes in the system. Finally, in AcTinG, colluders do not forward updates they received unofficially to their correct partners unless they also received them officially.

We varied the number of colluders, as well as the size of colluding groups. We measure the percentage of missed updates observed by correct nodes in presence of a proportion of colluders. We first studied the case in which all colluders belong to the same group. Results are depicted in Figure 7. The $\mathrm{X}$ axis presents the proportion of nodes that collude, while the $\mathrm{Y}$ axis presents the percentage of missed updates experienced by correct nodes in presence of colluders. We notice that correct nodes miss up to $98 \%$ of updates with BAR Gossip and $72 \%$ of updates with LiFTinG, whereas they do not miss any update with AcTinG.

We then studied the impact of spreading colluders in multiple independent groups. More specifically, we made several experiments in which we distributed $30 \%$ of all the nodes in colluding groups of identical size. We depict the results in Figure 8 . The $\mathrm{X}$ axis presents the size of colluding groups, while the $\mathrm{Y}$ axis presents the percentage of missed updates observed by correct nodes. We observe that spreading colluders in different groups has the same impact on the quality of the content downloaded by correct nodes.

\begin{tabular}{|l|c|c|c|c|c|}
\hline Group size & 2 & 4 & 8 & 10 & 50 \\
\hline Overhead $(\%)$ & 34.35 & 51.53 & 60.12 & 61.84 & 67.33 \\
\hline
\end{tabular}

TABLE I

OVERHEAD OF COLLUDERS IN ACTING.

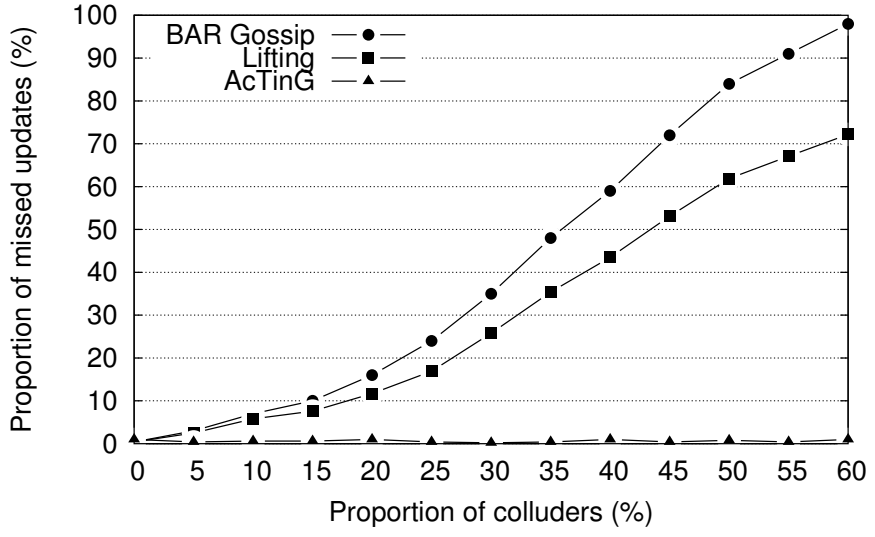

Fig. 7. Proportion of missed updates by correct nodes when a given proportion of the audience collude as a single group.

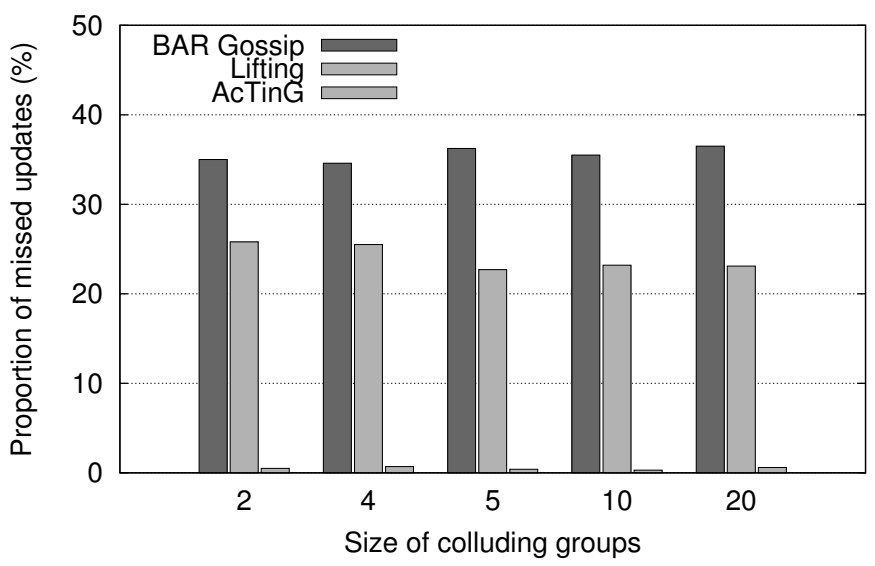

Fig. 8. Proportion of missed updates by correct nodes when $30 \%$ of the audience is rational, and collude in independent groups of equal sizes.

The reason why correct nodes do not observe missed updates when using AcTinG is that we designed AcTinG in such a way that colluders will eventually receive all the updates officially from their correct partners and will thus be obliged to forward them officially to their correct partners. Hence, engaging in a colluding group only yields an extra overhead due to the unofficial dissemination of updates among the group. We have measured this overhead and results are depicted in Table I. From this table we observe that the overhead due to collusion is at least of $34 \%$ of the size of the stream (case of a group containing only two colluders). In addition, as seen in section $\mathrm{V}$, in a scenario where $10 \%$ of nodes collude, and where audits are performed 5\% of the time, each deviation will be detected with a probability of $60 \%$. Moreover, exchanging updates without declaring them will provide at most a gain equal to $3 \%$. Consequently, nodes in AcTinG have no interest in colluding as they would not observe any increase in the quality of the stream they get, take a very high risk of being evicted, experience very low benefit, while suffering a useless waste of bandwidth.

\section{Bandwidth consumption}

To assess the overhead of AcTinG, we plot in Figure 9 the cumulative distribution of the average bandwidth consumption 
of nodes. Recall that AcTinG is used to broadcast a 300kbps stream. Figure 9 shows that AcTinG induces a reasonable overhead (that is mostly due to the transmission of logs). We also measured the memory consumption of AcTinG, which is due to the storage of secure logs and authenticators. Our measures have showed that a node consumes $3 \mathrm{MB}$ of memory for each partnership, in the worst case.

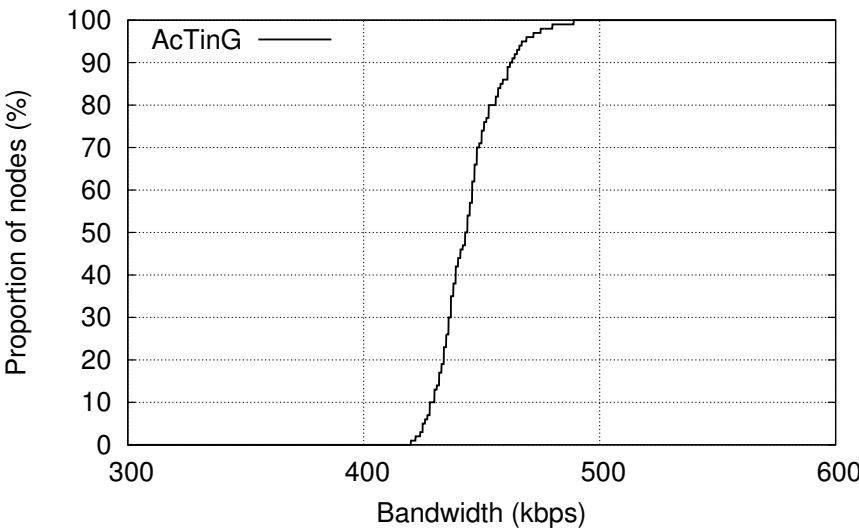

Fig. 9. Fault-free case: Cumulative distribution of average bandwidths.

\section{Resilience to massive node departure}

In the case of a massive node departure, the remaining nodes need to quickly replace their left partners with alive nodes in order not to miss updates. In this experiment, we measure the bandwidth consumption and the percentage of missed updates when $60 \%$ and $70 \%$ of nodes suddenly leave the streaming session. Results are depicted in Figures 10 and 11 respectively. Specifically, we observe in Figure 10 that the massive node departure, which happens 500 seconds after the beginning of the experiment, immediately causes a decrease in the average bandwidth consumed by the remaining nodes, as they stop exchanging messages with their left partners. This decrease (62\% and $75 \%$ in the case of the departure of $60 \%$ and $70 \%$ of nodes, respectively) is followed by an increase (of up to $18 \%$ and $27 \%$ in the former two cases), which corresponds to the messages exchanged by nodes to establish new partnerships (including a given proportion of audits). Finally, we observe that 30 seconds later, the average bandwidth consumption stabilises around $430 \mathrm{kbs}$ (13\% less than the original value), which is due to the decrease of the necessary number of partners per node.

We also compute the percentage of nodes that do not receive a viewable stream ${ }^{4}$. We observe in Figure 11 that only 2,5\% nodes do not receive a viewable stream during the first second when $60 \%$ nodes leave the system, and between $5 \%$ and $15 \%$ nodes do not receive a viewable during at most five seconds when $70 \%$ nodes leave the system.

\section{E. Scalability}

We performed simulations to evaluate the bandwidth, and the memory consumption, of AcTinG when the number of

\footnotetext{
${ }^{4}$ The stream is not viewable when more than $5 \%$ of the streaming windows cannot be displayed because of missed updates [7]
}

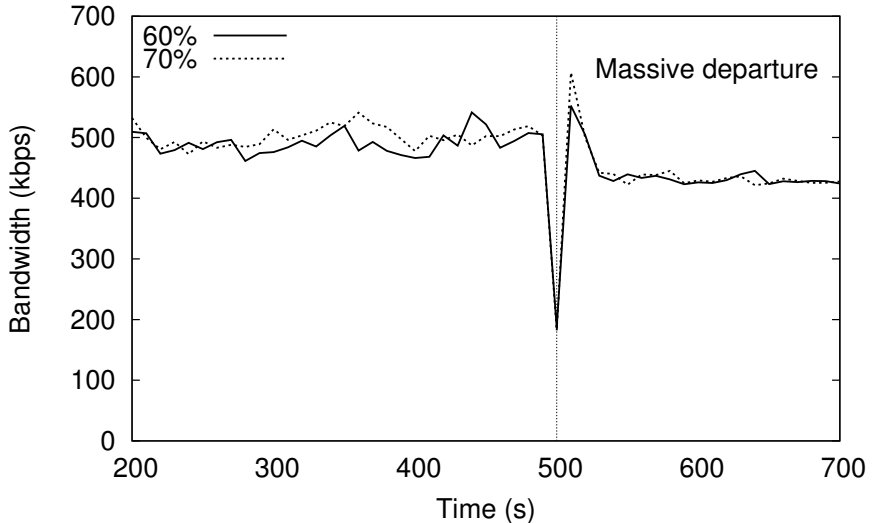

Fig. 10. Nodes average bandwidth after a massive departure.

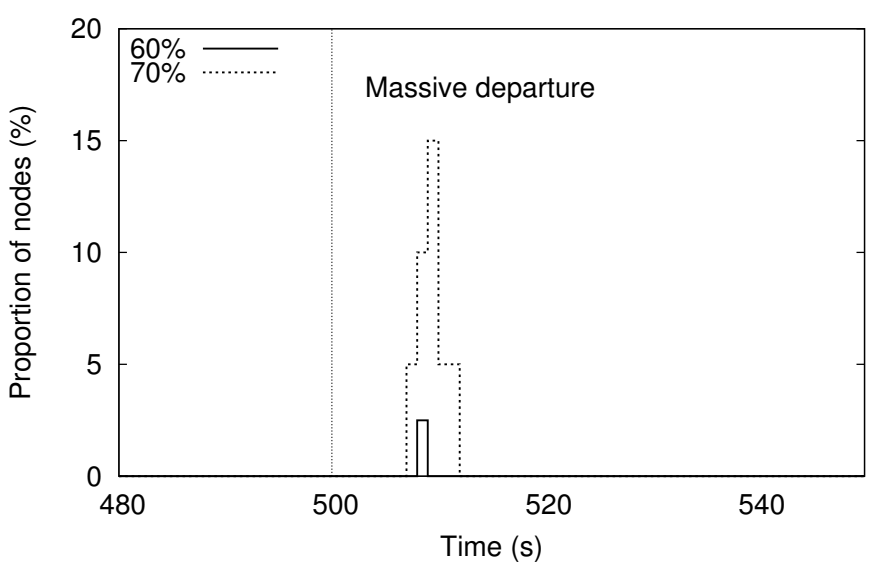

Fig. 11. Percentage of nodes that do not receive a viewable stream after a massive departure.

nodes increases in the system.

Results, depicted in Table II, show that both the bandwidth consumption and the memory consumption of AcTinG grow logarithmically with respect to the number of nodes in the system. Indeed, these values depend linearly on the number of partners a node has, which grows logarithmically with the system size.

\begin{tabular}{|r|c|c|}
\hline System size & $\begin{array}{c}\text { Bandwidth consumption } \\
(\mathrm{Kbps})\end{array}$ & $\begin{array}{c}\text { Memory usage } \\
(\mathrm{Mb})\end{array}$ \\
\hline 100 & 380.0 & 6.4 \\
500 & 436.6 & 9.5 \\
3,000 & 511.1 & 12.7 \\
22,000 & 603.4 & 15.9 \\
160,000 & 713.5 & 19.1 \\
$1,200,000$ & 841.4 & 22.3 \\
\hline
\end{tabular}

TABLE II

AVERAGE BANDWIDTH AND MEMORY USAGE OF ACTING IN FUNCTION OF THE SYSTEM SIZE.

\section{RELATED WORKS}

In this section, we focus on peer-to-peer content dissemination protocols that handle rational nodes. These protocols can be classified into two categories, according to the way file chunks (called updates in the following) are exchanged between nodes. The first category of protocols is composed 
of symmetric protocols. These protocols force nodes to collaborate, as the number of updates they get from a node is proportional to the number of updates they have to offer (this principle is often referred to as tit-for-tat). BAR Gossip [7] and FlightPath [22] are symmetric protocols relying on game theory. Both provide incentives to ensure that rational nodes respectively have no, or a limited, interest in deviating from the protocol. In terms of robustness to rational nodes, the BAR Gossip protocol exhibits stronger properties than the FlightPath protocol. Indeed, nodes in FlightPath are assumed to deviate only if the benefit they get is higher than a threshold, which is not the case in BAR Gossip. While the authors of these two protocols point out the problem of colluding rational nodes in [7], none of them address it.

The second category of protocols is composed of asymmetric protocols. These protocols require nodes to altruistically push update identifiers to other nodes, which subsequently pull updates of interest. A first protocol in that category is the one presented in [24]. This protocol aims at adapting the contribution of nodes to the systems, according to their available resources. This protocol assumes the existence of trusted auditors that run in dedicated external nodes and does not deal with colluders. A second protocol in that category is LiFTinG [8]. To the best of our knowledge, LiFTinG is the only existing peer-to-peer content dissemination protocol that tackles the problem of colluding rational nodes. Specifically, LiFTinG sporadically verifies the distribution of the interactions a given node performed with other nodes in the system. Nodes that collude with other nodes break the uniform distribution of partner selection, which may result in their detection. In order to be cost effective, LiFTinG only performs sporadic audits, and relies on non-secure logs that can contain wrong information, be incomplete, be tampered with and, as a consequence, be inconsistent the ones with respect to the others. As a result, LiFTinG suffers from two major limitations: correct nodes can be wrongly evicted from the system (false positives), and a proportion of colluding rational nodes can harm the system without being detected (false negatives).

\section{CONCLUSION}

A number of gossip-based content dissemination protocols tolerating rational behaviours have been proposed. A limitation of these protocols is that they do not handle rational nodes that collude, i.e. that act as a group in order to improve their benefit. The only exception is the LiFTinG protocol that performs sporadic checks on insecure logs to try to detect colluding nodes. We have shown in this paper that neither LiFTinG nor BAR Gossip, the most robust rational resilient content dissemination protocol, are effectively resilient to colluders. In this paper, we have presented AcTinG, the first content dissemination protocol that tolerates rational nodes acting both individually and in collusions, and that guarantees zero false positive accusations. Performance evaluation combining both a real deployment and simulations has demonstrated that nodes running AcTinG are able to deliver the entire content despite the presence of colluders. We have also shown that AcTinG is resilient to churn, and exhibits very desirable scalability properties with a logarithmic growth of memory and bandwidth consumption, comparable to standard gossip based protocols. Our future work includes the study of the applicability of the AcTinG principles to other types of collaborative applications for the accurate detection of rational (possibly colluding) nodes.

\section{ACKNOWLEDGEMENT}

Experiments presented in this paper were carried out using the Grid'5000 testbed, supported by a scientific interest group hosted by Inria and including CNRS, RENATER and several Universities as well as other organizations (see https://www.grid5000.fr).

\section{REFERENCES}

[1] E. Adar and B. A. Huberman, "Free riding on gnutella," First Monday, vol. 5, no. 10, 2000.

[2] R. Krishnan et al., "The impact of free-riding on peer-to-peer networks," in System Sciences, 2004. Proceedings of the 37th Annual Hawaii International Conference on. IEEE, 2004, pp. 10-pp.

[3] M. Feldman et al., "Free-riding and whitewashing in peer-to-peer systems," Selected Areas in Communications, IEEE Journal on, vol. 24, no. 5, pp. 1010-1019, 2006.

[4] J. F. e Oliveira et al., "Can peer-to-peer live streaming systems coexist with free riders?" in Peer-to-Peer Computing, 2013. P2P'13. IEEE 13th International Conference on, 2013.

[5] T. Locher et al., "Free riding in bittorrent is cheap," in Proc. Workshop on Hot Topics in Networks (HotNets). Citeseer, 2006, pp. 85-90.

[6] J. J.-D. Mol et al., "Give-to-get: free-riding resilient video-on-demand in p2p systems," in Electronic Imaging 2008, 2008.

[7] H. C. Li et al., "Bar gossip," in Proceedings of OSDI'06.

[8] G. et al., "Lifting: lightweight freerider-tracking in gossip," in Proceedings of Middleware'10.

[9] S. Ben Mokhtar et al., "Firespam: Spam resilient gossiping in the bar model," in Proceedings of SRDS, 2010.

[10] X. Vilaça et al., "N-party bar transfer," in Principles of Distributed Systems. Springer, 2011, pp. 392-408.

[11] L. Qiao et al., "An empirical study of collusion behavior in the maze p2p file-sharing system," in ICDCS'07.

[12] R. Eidenbenzet al., "Hidden communication in p2p networks steganographic handshake and broadcast," in INFOCOM, 2011 Proceedings IEEE. IEEE, 2011, pp. 954-962.

[13] L. Lamport et al., "The byzantine generals problem," ACM Trans. Program. Lang. Syst., vol. 4, no. 2, 1982.

[14] C. Ho et al., "Nysiad: practical protocol transformation to tolerate byzantine failures," in Proceedings of NSDI'08.

[15] A. Haeberlen et al., "Peerreview: practical accountability for distributed systems," SIGOPS Oper. Syst. Rev., vol. 41, no. 6, 2007.

[16] H. Andreaset al., "Accountable virtual machines," in Proceedings of OSDI'10.

[17] D. Levin et al., "Trinc: small trusted hardware for large distributed systems," in Proceedings of NSDI'09.

[18] C. Byung-gon et al., "Attested append-only memory: Making adversaries stick to their word," in Proceedings of SOSP'07.

[19] E. Patrick et al., "Epidemic information dissemination in distributed systems," IEEE Computer, vol. 37, no. 5, 2004.

[20] A. Aiyer et al., "Bar fault tolerance for cooperative services," in Proceedings of SOSP, 2005.

[21] T. Bressoud et al., "Hypervisor-based fault tolerance," ACM Transactions on Computer Systems (TOCS), vol. 14, no. 1, pp. 80-107, 1996.

[22] H. C. Li et al., "Flightpath: obedience vs. choice in cooperative services," in Proceedings of OSDI'08.

[23] S. Ben Mokhtar et al., "Acting: Accurate freerider tracking in gossip," University of Grenoble, Tech. Rep., 2014, https://sites.google.com/site/soniabm/.

[24] R. van Renesse et al., "Enforcing fairness in a live-streaming system," in Proceedings of MMCN'08. 